\title{
Lesões na Prática do Surfe
}

\section{Surfing injuries}

\section{Lesiones de surf}

\begin{abstract}
Resumo
A prática de surfe tem tido um grande destaque em todo o mundo. Seus adeptos têm aumentado de forma considerável, tornando-o o esporte náutico mais praticado atualmente. Por ser um esporte que requer grande esforço físico e mudanças bruscas de postura seus atletas estão sempre em grande risco de desenvolver lesões. Assim o objetivo desse estudo foi elencar as lesões que mais acometem os praticantes do surfe, bem como a conduta dos atletas para evitarem tais lesões e ainda compreender a importância do fisioterapeuta na prevenção e tratamento de lesões esportivas. A metodologia utilizada nesta pesquisa foi uma revisão de literatura, onde foram realizadas buscas literárias sobre este assunto em bases de dados eletrônica como Google Acadêmico, Scientific Eletronic Library Online (Scielo), PubMed, com o propósito de analisar e sintetizar as informações encontradas. Através deste estudo foi possível verificar as principais características das lesões que acometem os atletas do surfe. Sendo assim, é de grande valia o entendimento do gestual esportivo dessa modalidade e as condutas a serem tomadas com o intuito de minimizar o risco de lesões e visando os benefícios que promovam analgesia, melhora da flexibilidade, da força muscular, do condicionamento físico e cardiorrespiratório, constatando então que, o planejamento de um programa de prevenção de lesões com atletas do surfe, pode ser considerado um excelente método para redução de lesões.
\end{abstract}

Palavras-chave: Surfe; Esportes aquáticos; Lesões esportivas; Fisioterapia.

\begin{abstract}
The practice of surfing has been highlighted all over the world. Its practitioners have increased considerably, making it the most practiced water sport today. Because it is a sport that requires great physical effort and sudden changes in posture, your athletes are always at great risk of developing injuries. Thus, the objective of this study was to list the injuries that most affect surfers, as well as the conduct of athletes to avoid such injuries and also to understand the importance of the physiotherapist in the prevention and treatment of sports injuries. The methodology used in this research was a literature review, where literary searches were carried out on this subject in electronic databases such as Google Scholar, Scientific Electronic Library Online (Scielo), PubMed, with the purpose of analyzing and synthesizing the information found. Through this study it was possible to verify the main characteristics of the injuries that affect surfing athletes. Therefore, understanding the sports gestures of this modality and the conducts to be taken in order to minimize the risk of injuries and aiming at the benefits that promote analgesia, improve flexibility, muscle strength, physical and cardiorespiratory fitness is of great value, realizing that planning an injury prevention program with surfing athletes can be considered an excellent method for to reduce the number of injuries.
\end{abstract}

Keywords: Surf; Water sports; Sports injuries; Physiotherapy.

\section{Resumen}

La práctica del surf se ha destacado en todo el mundo. Sus practicantes han aumentado considerablemente, convirtiéndose en el deporte acuático más practicado en la actualidad. Por ser un deporte que requiere un gran esfuerzo físico y cambios bruscos de postura, tus deportistas siempre corren un gran riesgo de desarrollar lesiones. Así, el objetivo de este estudio fue enumerar las lesiones que más afectan a los surfistas, así como la conducta de los deportistas para evitar tales lesiones y también comprender la importancia del fisioterapeuta en la prevención y tratamiento de las lesiones deportivas. La metodología utilizada en esta investigación fue una revisión de la literatura, donde se realizaron búsquedas literarias sobre este tema en bases de datos electrónicas como Google Scholar, Scientific Electronic Library Online (Scielo), PubMed, con el propósito de analizar y sintetizar la información encontrada. A través de este estudio fue posible verificar las principales características de las lesiones que afectan a los deportistas surfistas. Por tanto, es de gran valor comprender los gestos deportivos de esta modalidad y las conductas a tomar con el fin de minimizar el riesgo de lesiones y apuntar a los beneficios que favorezcan la analgesia, mejoren la 
flexibilidad, la fuerza muscular, la aptitud física y cardiorrespiratoria. , al darse cuenta de que planificar un programa de prevención de lesiones con deportistas de surf puede considerarse un método excelente para reducir las lesiones.

Palabras clave: Surf; Deportes acuáticos; Lesiones deportivas; Fisioterapia.

\section{Introdução}

O surfe tem sua origem por volta do século XI, quando o capitão de origem inglesa James Cook chegou as ilhas do Pacífico por volta de 1778 e lá avistou os primeiros surfistas, sendo o primeiro europeu a presenciar e escrever sobre o surfe. Nos diários escritos pelo inglês, ele relatou que o surfe era considerado imoral, pois os nativos andavam seminus e porque tinha um caráter lúdico, além de representar a ociosidade e a liberdade (Kampion, 2003). Em consequência disso, a prática do surfe desapareceu por volta do século XIX.

O surfe ressurgiu por volta de 1900 a 1945, em franca expansão devido a evolução do material utilizado e com maior número de praticantes (Carozza, 2013). Assim, o surfe começou a ser difundido pelo mundo e a partir da década de 60 se deu início às instituições de surfe pelo planeta, e também o início das competições e do profissionalismo do esporte. No Brasil, foi por volta de 1938 que os primeiros praticantes despontaram, na praia do Gonzaga, no litoral de Santos. Mas, somente em 1947 que o surfe começa a se expandir para as praias do país, com o surfista Luís Carlos Vital na praia do Arpoador na cidade Maravilhosa. Contudo, somente na década seguinte que o surfe começou a ganhar força no Rio de Janeiro (Lorch, 1980; Semes, 1997).

Atualmente, os adeptos tem crescido consideravelmente no Brasil, e devido a existência de um longo litoral e com condições favoráveis à pratica do esporte, como ondas grandes e, juntamente com a conquista de um campeonato mundial por um surfista brasileiro, talvez tenha sido um fator para esse aumento de número de praticantes, tanto amadores como profissionais (Steinman et al., 2000). A International Surf Association relata que há quase 17 milhões de praticantes do surfe no mundo, e o Brasil está entre os três grandes do surfe no mundo. O surfe é uma modalidade esportiva que requer um bom preparo físico, visto que durante sua prática o atleta muda bruscamente suas posições exigindo um grande equilíbrio e esforço muscular. Além disso, o esporte faz exigências aos atletas, como condicionamento para suportar o impacto das ondas e efeitos de grande magnitude no seu desempenho, e gerar a execução de movimentos ininterruptos e intermitentes (Vretaros, 2015).

Destaca-se que, além das lesões causadas pelos movimentos durante a pratica do esporte, outras lesões podem ser causadas dependendo do tamanho da onda a ser surfada, do tipo do fundo do mar bem como o impacto contra a prancha que pode levar a lacerações (Base, 2007). Estudos relatam uma taxa de 6,6 lesões a cada 1000 horas de surfe, e que a quantidade de lesões foi maior em atletas que surfavam em grandes ondas ou em mares com corais no fundo (Nathanson, Bird, Dao e Tam-Sing, 2007).

Como esse esporte vem crescendo diariamente, diversos autores vêm pesquisando formas de evitar as lesões. Os incidentes de lesões em atletas do surfe pode se dar devido a postura de hiperextensão da coluna lombar e também da cervical que o atleta tem durante a atividade (Steinman et al., 2000). Uma maneira de minimizar essas tais é o fortalecimento da musculatura da região do abdome e de treinos específicos da modalidade, pois, geralmente os atletas não possuem um treinamento específico com especialistas desportivos, não tendo treinamentos adequados que visam diminuir e minimizar o risco de lesões durante o esporte, ocasionando tais lesões (Danucalov, Ornellas e Navarro, 2009).

Mesmo o surfe sendo um esporte em grande expansão e ascensão, não são encontrados na literatura um número expressivo de estudos que relatem as lesões oriundas desse esporte. Com base nos pressupostos acima, o presente estudo adota, elencar as lesões que mais acometem os praticantes do surfe, bem como a conduta terapêutica no campo da fisioterapia. 


\section{Metodologia}

Trata-se de estudo de revisão de literatura sobre a importância de programas de prevenção de lesão para praticantes de surfe, no qual realizou-se analise de conteúdo da temática. A busca das informações cientificas disponíveis na literatura ocorreu nas bases de dados eletrônicos Scielo, Google Acadêmico e Pubmed. Foram investigadas publicações entre os anos de 1997 a 2019.

Após o levantamento bibliográfico, procedeu-se ao ordenamento e analise das publicações selecionadas, com o intuito de analisar e discutir os principais aspectos relacionados à temática, apresentados pela literatura.

\section{Resultados e Discussão}

\subsection{Lesões mais frequentes no surfe}

São consideradas lesões esportivas, qualquer problema médico ocorrido durante a prática esportiva, que pode levar o atleta a perder parte ou todo treinamento e competição ou limitar sua habilidade atlética. (Gantus e Assumpção, 2002), Além do mais, todo esporte tem em si a capacidade de produzir lesões, e no surfe, essas lesões podem se manifestar de forma musculo esqueléticas, fraturas, contusões, dentro outras (Meneses, 1983; Vretaros, 2015).

Em um estudo realizado com 32 surfistas profissionais avaliando a incidência de lesões desse esporte, encontraram, 112 lesões diferentes, estando os cortes entre as lesões de maior numero (33,9\%), seguido das entorses (25,9\%), também encontraram $14,2 \%$ de contusões bem como $12,5 \%$ de casos de estiramentos. Os surfistas também relataram casos de queimaduras $(8 \%)$ e fraturas $(5,3 \%)$. Com essa pesquisa observaram que o choque com a prancha ou com o fundo do mar são os principais causadores de tais lesões, tendo algumas manobras realizadas pelos atletas certa responsabilidade em determinadas lesões relatadas (Base, Alves, Martins e Costa, 2005)

Na pesquisa realizada por Nathanson et al., (2007), onde adquiriram uma amostra de 15.645 surfistas que participaram de campeonatos entre os anos de 1999 e 2005 os autores relatam que a cada 1.000 surfistas, 5,7 apresentam risco de alguma lesão. Eles relataram 116 lesões, sendo a entorse do joelho a de maior frequência. Em tal pesquisa os autores relataram também que a possibilidade de lesão foi aproximadamente 2 vezes maior quando os praticantes estavam surfando em mar com grandes ondas e quase 3 vezes maior quando o fundo do mar era de pedra.

$\mathrm{Na}$ pesquisa desenvolvida por Taylor et al., (2004) os autores analisaram as lesões que ocorreram nos 12 meses prévios. Os entrevistados foram na maioria homens com media de idade de 27 anos e que já praticavam o surfe há pelo menos 10 anos. Eles observaram que as lesões de maior frequência são fraturas, lacerações e os estiramentos, e essas lesões atingem principalmente a cabeça e os membros inferiores. O choque com a prancha, ou com o fundo do mar e até mesmo o choque com outro atleta foi relatado como as principais causas do acontecimento de lesões.

Em 2010, Júnior e Ornellas, apontaram que boa parte das lesões são ocasionadas pela postura que o atleta desenvolve na remada sobre a prancha e não enquanto executa as manobras. Os autores trazem como causa dessas lesões, o fato de que os surfistas não realizam treinamentos específicos para a prática do surfe e também não fazem aquecimento ou algum tipo de alongamento antes de entrar no mar.

Steimman et al., (2000), descreveram que a maior parte das lesões acometem os membros inferiores. Casos de cortes foram mais encontrados nas pernas e pés, mas também foram apontados um número considerável de corte nos braços dos atletas, isso se deve, provavelmente, a esse membro estar em grande contato com a prancha durante a queda. Como resultado dessa pesquisa, observou-se que as distensões musculares e as contusões, em sua grande maioria ocorrem nos membros inferiores, no caso de lesões na cabeça, o nariz e o crânio são as partes mais atingidas. Quando se trata de lesões ligamentares o estudo aponta o joelho como o local mais acometido seguido do tornozelo. Casos de fratura foram pouco encontrados nesse estudo e quando relatados o joelho foi o mais acometido por tal lesão. Já os casos de luxações foram em sua maioria no ombro. 
Nessa pesquisa alguns casos de queimaduras por água vivas foram relatados pelos surfistas. Afonso et al., (2020) a afirma em seu estudo que lesões desportivas possuem relação direta com o tipo de esporte praticado e, geralmente, acometem membros, principalmente inferiores, articulações e grupos musculares mais usados durante a pratica esportiva.

\subsection{Prevenção de lesões na prática esportiva}

Em qualquer prática esportiva, coletiva ou individual, incluindo a prática do surfe o atleta está sujeito a se lesionar, algumas lesões ocorrem por traumas, outras por realização de movimentos repetitivos ou até mesmo por sobrecarga, e em esportes de alta velocidade podem ser ocasionadas pela mudança brusca de direção. Como algumas dessas lesões podem acarretar em afastamento temporário do esporte ou, dependendo do grau dessa lesão, ate mesmo interrupção permanente da pratica esportiva.

Um dos principais modelos teóricos para prevenção de lesões desportivas foi criado no inicio dos anos 90 e ele está dividido em 4 etapas. A primeira dela é estabelecer a prevalência e incidência das lesões, a segunda é desvendar a etiologia e mecanismo das lesões seguida da implementação de uma intervenção para a prevenção das lesões e por fim, mensurar a efetividade da intervenção proposta.

Finch (2006) aponta que um importante fator para aumentar a eficácia de programas de prevenção de lesões esportivas é saber o que os profissionais que atuam com esporte pensam sobre lesão e sua prevenção, pois assim se tem uma melhor adequação das intervenções propostas assim como na escolha de quais estratégias serão adotadas para a implementação do programa proposto.

Hall \& Brody (2001) relatam que os exercícios de alongamentos são os mais realizados pelos atletas pois eles aumentam a extensibilidade musculotendínea e do tecido conjuntivo muscular e periarticular, contribuindo para aumentar a flexibilidade. Existem basicamente três métodos de alongamento: o alongamento passivo, o ativo e a facilitação neuromuscular proprioceptiva (FNP). Apesar do método FNP ser o mais eficaz, o alongamento estático é mais utilizado, pois é o método mais seguro, simples e com menor risco de lesão.

Segundo Nathanson et al., (2007), o surfista deve fazer algum tipo de aquecimento previamente antes de entrar na água para a pratica do surfe. Segundo esses autores, o atleta também deve realizar atividades de alongamento, logo após terminar a prática esportiva diminuindo assim a chance de desenvolver algum tipo de lesão.

Em um estudo desenvolvido por Pope, Hebert, Kirwan e Grahan (2000) onde 2 grupos de homens foram estudados durante 3 meses, os autores analisaram se o alongamento seria realmente eficaz para diminuir lesões nos membros inferiores. Durante esse período, os dois grupos realizaram aquecimento antes das atividades, no entanto apenas um grupo fez o alongamento ao término do treino. Como resultados dessa pesquisa não foram encontrados efeitos significativos do alongamento para prevenir lesões, tanto dos tecidos moles quanto ósseo. Os autores sustentam que a incidência de lesão está ligada a idade e ao nível de condicionamento físico, e não à prática de alongamento. Dentre as características adquiridas com o condicionamento físico pode-se citar, maior recrutamento neuromuscular de fibras, maior capacidade das fibras de absorver energia e transferi-la para o sistema ósseo e maior quantidade de substrato energético. E, no entanto, a prática de alongamento não resulta na obtenção destes fatores.

No entanto, Amako et al., (2003) realizaram um estudo onde analisaram o efeito do alongamento estático sobre a prevenção de lesão em recrutas militares. Foram divididos em 2 grupos, o primeiro grupo realizou um exercício de alongamento antes e após o treinamento físico, e o segundo grupo realizou apenas o programa de treinamento físico. Os resultados mostram que o grupo 1 , que foi submetido ao alongamento, teve uma incidência menor de lesões musculoesqueléticas quando comparado ao grupo que não foi alongado. A eficácia do alongamento na prevenção de lesões ainda é pouco conhecida, e fatores como idade, tipo de alongamento, frequência de treino, duração, e intensidade devem ser 
considerados na prevenção de lesões. Desta forma, há pouca evidência sobre a relação entre o aumento de flexibilidade e a redução da incidência de lesão (Emery et al., 2007).

Domingues e Coimbra (2008) relatam em seu estudo que um treinamento proprioceptivo pode ser utilizado para a recuperação cinético funcional bem como para prevenir lesões no esporte. Os autores relatam também que a reeducação proprioceptiva após uma lesão contribuiu muito para a perda de medo no retorno a prática do esporte.

Corforme Holm et al., (2004) os exercícios específicos de propriocepção levam o corpo a responder com maior eficácia, melhorando a força, a coordenação motora, o equilíbrio, tempo de reação a determinadas situações e compensar a perda de sensações ocasionadas por uma lesão articular. No estudo realizado por Base et al., (2007) onde apontam os traumas e cortes como lesões frequentes entre os praticantes do surfe os autores sugerem que os surfistas devem optar usar sempre pranchas de materiais mais modernos, que são mais leves por exemplo, diminuindo assim o trauma com o equipamento.

Para evitar o afastamento temporário ou até mesmo permanente do esporte o melhor a se fazer é prevenção, porem de acordo com o estudo feito por De Moraes, Guimarães e Gomes (2013) nenhum dos surfistas participantes obteve afastamento da prática do surfe por conta das lesões e que a maior parte fez tratamento com medicamentos, alguns com alongamentos e exercícios e a minoria com crioterapia e curativos. Um estudo desenvolvido por Steinman et al., (2000) demonstrou que boa parte dos praticantes de surfe não procuram atendimento médico imediatamente após a ocorrência da lesão, eles demoram em média até 14 dias para tomar alguma providência em relação as suas lesões e que os casos em que procuraram atendimento de um profissional da saúde foi de lesões do tipo ligamentar ou da coluna vertebral na região lombar. Nesse estudo também foi revelado que a maior parte dos surfistas enfrenta lesões músculo esquelética por falta de preparo muscular, falta de força e treinamento, movimentos repetidos, posição inadequada e desequilíbrio muscular.

Conforme a literatura pesquisada averiguou-se que é necessário identificar o tipo de esporte, frequência, intensidade de exercício, fatores de risco e o mecanismo de lesão para que se possa adotar uma medida ou programa para prevenção de lesões em tais esportes.

\subsection{Abordagem fisioterapêutica na prevenção e tratamento de lesões esportivas}

A fisioterapia desportiva é focada na prevenção de lesões, onde o profissional fisioterapeuta baseado na biomecânica do exercício, na fisiologia da ação muscular, articular e sistêmica geral, além da anatomofisiologia, e na intensidade dos treinamentos elabora um programa de treinamento para executar com os atletas. Já nos casos em que se teve lesões a atuação deve ser o mais precoce possível para o atleta poder retornar a suas atividades esportivas, daí a importância do fisioterapeuta no meio esportivo. Afonso et al., (2020) ${ }^{\mathrm{b}}$ destaca que o fisioterapeuta atua nas equipes técnicas de treinamento, e contribui com a avaliação, programação, promoção e execução da reabilitação dos atletas nas mais variadas modalidades desportivas, e também na elaboração de programas preventivos, que objetivam minimizar a ocorrência de lesões.

De acordo com Fontana (1999), o objetivo da fisioterapia é restabelecer a função que é perdida diante de uma lesão, atuando também na prevenção, gerando através de um treinamento especifico a potencialização do desempenho esportivo do atleta. $\mathrm{O}$ autor destaca assim a importância de um fisioterapeuta na equipe de treinos desses indivíduos.

Prati (1992), afirma que o fisioterapeuta deve estar integrado na equipe multidisciplinar desportiva bem como conhecer o esporte, metodologia usada pelo treinador dos atletas e compreender os mecanismos das lesões. Além do mais, o profissional da fisioterapia deve conhecer a mecânica esportiva, a fisiologia da ação muscular e também ter conhecimento da área terapêutica quando esta for necessária. O fisioterapeuta deve desenvolver técnicas de alongamento, facilitação neuromuscular, exercícios de propriocepção bem como exercícios de fortalecimento muscular.

Kloetzel (1985) descreve que grande parte das lesões no surfe se deve ao excessivo uso dos músculos sem devida prevenção e recuperação. $\mathrm{O}$ autor afirma que deve-se fazer um trabalho nos músculos do tronco e do abdômen, nos membros 
inferiores e superiores e na região da cabeça e pescoço dos surfistas objetivando a melhora da força, flexibilidade e também os reflexos musculares. Esse trabalho pode ser realizado por um fisioterapeuta e incluem atividades de alongamento, fortalecimento e exercícios proprioceptivos bem como exercícios pliométricos.

A pliometria é uma técnica que foi desenvolvida nos anos 60 onde treinadores norte americanos usavam saltos com bancos e o pular corda em seus treinamentos, porém não conheciam sua base fisiológica. No final da década de 60 o treinador soviético Yuri Verkhoshanski, começou a transformar esses saltos aleatórios, em treinamento pliométrico organizado. Essa técnica só recentemente começou a ser usada para a prevenção e recuperação de lesões visto que ela gera um aumento na potencia muscular aumentando assim o rendimento dos atletas. Os exercícios pliométricos se utilizam do ciclo alongamentoencurtamento (CAE), eles ativam o ciclo excêntrico concêntrico do músculo esquelético, durante os exercícios pliométrico é aproveitado o potencial elástico acumulado durante ações excêntricas e liberado posteriormente na fase concêntrica sob a forma de energia cinética, aumentando a produção de força com menor custo metabólico. Por essa técnica ser capaz de aumentar a resposta muscular e melhorar a coordenação neuromuscular bem como desenvolver força explosiva ela é utilizada na fase final da reabilitação de lesões musculoesqueléticas, e também na prevenção de alguns tipos de lesões.

Andrews, Harrelson e Wilk (2000) em seu estudo apontam os exercícios pliométricos como sendo bem eficazes no tratamento para reabilitar atletas com síndrome do impacto, instabilidade de ombro, lesões do cotovelo e no pós-cirúrgico de lesões do lábio glenoidal. Os autores defendem também a utilização dessa técnica para tratamento de lesões do membro inferior, como por exemplo as tendinopatias, lesões musculares, entorses de tornozelo, lesões ligamentares.

De acordo com Bompa (2004) o fisioterapeuta deve analisar os movimentos específicos de cada esporte avaliando variáveis como intensidade, frequência e sentido do movimento criando assim um programa de exercícios pliométricos de acordo coma necessidade do atleta conseguindo assim obter um resultado eficaz. Segundo Howard (2004) o atleta que será submetido a uma reabilitação usando da pliometria deve estar com ausência de dor e edema, com a amplitude do movimento normal e força muscular similar ao membro contralateral, justificando com isso o motivo dessa técnica ser adotada no final da reabilitação. Os exercícios pliométricos também são contraindicados nos pós-operatórios imediatos e derrame articular. Não há consenso quanto ao volume, intensidade e frequência que os exercícios pliométricos devem ser realizados na fase de reabilitação dos atletas porem Howard (2004) cita que as sessões não devem ser realizados em dias seguidos e no inicio deve ser feitos exercícios mais simples e de baixa intensidade e progressivamente ir aumentando para exercícios mais complexos e de maior intensidade.

De acordo com Wilk et al., (1993) exercícios pliométricos não devem ser realizados mais que 3 vezes na semana. Como a pliometria pode causar fadiga muscular o tempo de repouso entre as sessões é muito importante de ser seguido, visto que se o controle motor ficar comprometido devido a fadiga os efeitos do treinamento pliométrico nãos serão alcançados.

Como cita Deslandes, Gain, Hervé e Hignet (2003) o fisioterapeuta deve orientar ao atleta o correto posicionamento corporal durante a pratica de exercícios envolvendo a pliometria. Os autores relatam ainda que o não cumprimento do repouso entre as sessões, bem como a aplicação de exercícios pliométricos no inicio do tratamento das lesões podem piorar as mesmas ou ainda causar novas lesões.

\section{Considerações Finais}

Segundo a bibliografia consultada para esta revisão literária, constatou-se que as lesões predominantes em praticantes do surfe variam conforme a localidade, em praias com ondas grandes e a entorse do joelho foi a lesão mais encontrada. Já no Brasil, onde as ondas são de menor porte, predominaram os ferimentos corto-contusos e as lacerações. Outra questão que necessita de investigação são os diferentes tipos de lesões que ocorrem em surfistas amadores e profissionais. Visto que, para 
se sagrar campeão, o atleta realiza manobras mais complexas se expondo a um maior risco deter algum tipo de lesão, bem como um surfista profissional pratica o esporte em uma quantidade maior que o surfista amador.

Em relação a prevenção das lesões no surfe, averiguou-se que atletas profissionais que realizam treinamento funcional com objetivo de minimizar diminuir a probabilidade de desenvolverem alguma lesão, bem como ter um melhor desempenho nas competições, alguns relataram realizarem exercícios de musculação, tendo o pilates e a corrida sido mencionados como exercícios praticados pelos mesmos. Em alguns estudos foi relatado que exercícios de propriocepção devem ser realizados por atletas para prevenir lesões.

Porém, há umas grandes diferenças de opiniões e achados bibliográficos referentes a essa prevenção. Alguns estudos relatam a eficácia do alongamento na prevenção de lesões, outros não acharam diferenças estatísticas entre grupos que fizeram ou não o alongamento antes da atividade física. Alguns autores relatam que fatores como idade, tipo de alongamento, frequência de treino, duração e intensidade devem ser considerados na prevenção de lesões. Necessita-se de mais estudos científicos de alta qualidade, para averiguar os efeitos do alongamento para prevenção de lesão nos diferentes tipos de esporte, incluindo o surfe.

Foi encontrada na literatura também os exercícios pliométricos como uma possível técnica para ser usada tanto na prevenção quanto na reabilitação das lesões no esporte. Porem por ser uma técnica relativamente nova neste aspecto existe ainda a necessidade de mais estudos que comprovem os benefícios da pliometria na reabilitação das lesões. Entretanto é de grande importância que o profissional da fisioterapia conheça essa técnica bem como sua aplicação clinica na prevenção e tratamento das lesões para que assim possa criar um programa eficiente em todos os aspectos visto que os exercícios dessa natureza podem ser realizados em qualquer ambiente e de uma forma bem simples.

Conclui-se assim que ainda há poucas referências no que diz respeito a prevenção de lesões especificamente entre os praticantes do surfe bem como seus possíveis tratamentos. Salienta-se com essa revisão a importância da fisioterapia esportiva, que é aquela voltada para atletas, que tem como função prevenir e proteger os mesmos de lesões causadas durante a prática do exercício bem como permitir a recuperação dos atletas quando estes são acometidos por algum problema. Os fisioterapeutas atuam em seus consultórios particulares, em ambientes desportivos bem como no Sistema Único de Saúde, onde podem executar seus serviços em duas estruturas distintas que são os ambulatórios de especialidades e a NASF (Núcleo ampliado de saúde da família). O acompanhamento de um atleta por um fisioterapeuta permite que o profissional identifique previamente algum distúrbio ou alterações que possam a vir a prejudicar o atleta diminuindo seu rendimento no esporte.

\section{Referências}

Afonso, M. dos S., Sousa, W. W. da S. e, Afonso, M. dos S., Junior, A. de O. S., Neves, F. B., \& Lourenção, L. G. (2020). ${ }^{a}$ Análise das lesões em atletas amadores praticantes de corridas de rua. Research, Society and Development, 9(3), e101932614. https://doi.org/10.33448/rsd-v9i3.2614

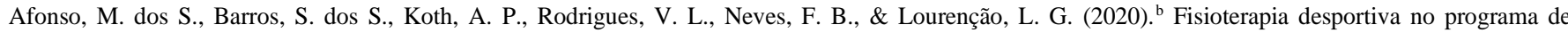
prevenção de lesão no futebol profissional. Research, Society and Development, 9(3), e72932434. https://doi.org/10.33448/rsd-v9i3.2434

Amako, M., Oda, T., Masuoka, K., Yokoi, H., Campisi, P. Effect of static stretching on prevention of injuries for military recruits. Mil Med. 2003;168(6):4426 .

Andrews, J. R., Harrelson, G.L., Wilk, K. E. Reabilitação física nas lesões desportivas. (2a ed.), Guanabara Koogan, 2000.504.

Base, L. H., Alves, M. A. F., Martins, E. O., Costa, R. F. Lesões em surfistas profissionais. Rev Bras Med Esporte. 2007;13(4):251-3.

Bompa, T. O. (2004). Treinamento de potência para o esporte. Phorte, 193

Carozza, M. Respostas fisiológicas à prática do surf recreativo em adultos. Dissertação (Mestrado) - Universidade Lusófona de Humanidades e Tecnologias, Faculdade de Educação Física e Desporto. Lisboa - Portugal, 2013.

Danucalov, M. Á., Ornellas, F. H., \& Navarro, F. Força muscular isocinética, perfil de surfistas brasileiros. Revista Brasileira de Ciência e Movimento. Vol. 14. Núm. 4. p.78-82. 2009.

De Moraes, C. G., Guimarães, T. B. A., \& Gomes, R. S. A. Análise da prevalência de lesões em surfistas do litoral paranaense. Acta Ortop Bras. 2013;21(4):213-8 
Deslandes, R., Gain, H., Hervé, J. M., \& Hignet, R. Princípios de fortalecimento muscular: aplicaciones en El desportista. IN: Simonnet, J. Kinesioterapia. Medicina física. Paris: Elsevier, 2003:1 -10.

Domingues, M. P. L., \& Coimbra, F. C. D. E. F. Treino proprioceptivo na prevenção e reabilitação de lesões nos jovens atletas. Rev Desporto Saúde. 2008; 4(4):29-37.

Emery, C. A., Rose, M. S., Sarah, M., Mcallister, J. R., \& Meeuwisse, W. H. A prevention strategy to reduce the incidence of injury in high school basketball: a cluster randomized controlled trial. Clinical Journal of Sport Medicine. 2007; 17(1):17-24.

Finch C. A new framework for research leading to sports injury prevention. J Sci Med Sports 2006;9(1-2):3-9.

Fontana, R. F. (1999). O papel da fisioterapia na performance do atleta. In: simpósio internacional de fisioterapia. Revista Fisioterapia Universidade São Paulo, 6, 24.

Gantus, M. C., Assumpção, J. D. (2002). Epidemiologia das lesões do sistema locomotor em atletas de basquetebol. Acta Fisiátrica, São Paulo, 9(2), 7784.

Hall, M. C., Brody, T. L. (2001). Exercícios terapêuticos: na busca da função. Guanabara Koogan.

Holm, I., Fosdahl, M. A., Friis, A., Risberg, M. A., Myklebust, G., Steen, H. (2004). Effect of neuromuscular training on proprioception, balance, muscle strength, and lower limb function in female team handball players. Clin J Sport Med. 14(2):88- 94.

Howard, L. (2004). Plyometric concepts reinvent lower extremity rehabilitation. Biomechanics.

Júnior, N. N., \& Ornellas, F. H. (2010). Características do treinamento e lesões em surfistas amadores. Dissertação para o grau de Pós-Graduado e Bacharel. Universidade Federal de Santa Catarina, Florianópolis. pp. 27

Kampion, D. (2003). Stoked: A History of Surf Culture. Gibbs Smith.

Kloetzel, K. (1985). O que é Medicina Preventiva. Brasiliense.

Lorch, C. K. (1980). Surfe: Deslizando Sobre as Ondas. Guanabara Dois.

Meneses, L.J.S. (1983). O esporte e suas lesões. Rio de Janeiro: Palestra Edições Desportivas.

Nathanson A, Bird S, Dao L, \& Tam-Sing K. (2007). Competitive surfing injuries: a prospective study of surfing-related injuries among contest surfers. Am J Sports Med. 35(1):113-7.

Pereira, A. S. et al. (2018). Metodologia da pesquisa científica. UAB/NTE/UFSM. https://repositorio.ufsm.br/bitstream/handle/1 /15824/Lic_Computacao_Metodologia-Pesquisa-Cientifica.pdf?sequence=1

Pope, R. P., Hebert, R. D., Kirwan, J. D., \& Grahan, B. J. (2000). A randomized trial of preexercise stretching for prevention of lower-limb injury. Med. Sci. Sports Exerc.32(2):271-7.

Prati, F. A. M. (1992). Lesão: fatores desencadeantes e prevenção. Revista Fisioterapia em Movimento, 5(1), 9-20.

Secretaria Municipal De Santos A História do Surfe em Santos. Caderno SEMES. (vol.3). Santos: Autor, (1997).

Steinman, J., Vasconcelos, E. H., Ramos, R. M., Botelho, J. L., \& Nahas, M. V. Epidemiologia dos acidentes no surfe no Brasil. Rev Bras Med Esporte. 2000;6(1):9-15.

Taylor, D. M., Bennett, D., Carter, M., Garewal, D., Finch, C. F. (2004). Acute injury and chronic disability resulting from surfboard riding. J Sci Med Sport. 7(4):429-37.

Vretaros, A. (2015). Futebol: bases cientificas da preparação de força. Edição do autor.

Wilk, K. E., Voight, M. L., Keirns, M. A., Gambetta, V., Andrews, J. R., \& Dillman, C. J. (1993). Stretch-shortening drills for the upper extremities: theory and clinical application. J Orthop Sports Phys Ther. 